\title{
An Intelligent Intensive Care Unit for Patient Monitoring System
}

\author{
Manasa I R, Pallavi M, Priyanka S G, Suma B, Madhu M Nayak* \\ Department of CSE, GSSSIETW, Mysuru, Karnataka, India.
}

DOI: https://doi.org/10.21467/proceedings.1.13

* Corresponding author email: madhu.m@gsss.edu.in

\begin{abstract}
In the project, An Intelligent intensive care unit patient monitoring system is to implement ICU management system in order to reduce the fatality cases caused due to miscommunication and failed follow up among paramedical staff and doctors. The scope of the project is maintaining the temperature of the ICU and the patient and monitoring the pulse rate/heart beat and humidity. Regular monitoring of patients by sensors through push messages to the nurse. Authorized person can only view/access the prescription. One-time password is used for security purpose while accessing the message.
\end{abstract}

Keywords: Wearable sensor, Zigbee, deep learning, optimization.

\section{INTRODUCTION}

In India, Health care industry has undergone a cutting-edge revolution in terms of drugs, treatment and surgery. The main concept of the project is to monitor the patient continuously to avoid from critical situation. Hospitals have adopted high end hospital management system software. They have actively adopted information technology in terms of patient's database and transfer of the information to the doctors through network. The project is being specially designed for temperature, humidity, pulse rate is an important parameter to asses the critical status of the patient who has in ICU. The push notifications send to the paramedical staffs, it helps to monitoring the patient. Design a secure information transmission using MQTT message passing protocol. MQT'T protocol is used with low bandwidth and battery power to collect data for server. Build an interactive Android application by integrating all the features. The parameters values are going to abnormal it will automatically send alert mail to the doctors and relatives. Human beings normal body temperature is $35^{\circ} \mathrm{C}$. The temperature value is going to $40^{\circ} \mathrm{C}$ the mail is sent. Normal heart rate is 72 , if it goes to 85 it will automatically send mail. At the same respiration rate is goes to 40 it will automatically send mail to the doctors and relatives.

(C) 2018 Copyright held by the author(s). Published by AIJR Publisher in Proceedings of the $3^{\text {rd }}$ National Conference on Image Processing, Computing, Communication, Networking and Data Analytics (NCICCNDA 2018), April 28, 2018.

This is an open access article under Creative Commons Attribution-NonCommercial 4.0 International (CC BY-NC 4.0) license, which permits any non-commercial use, distribution, adaptation, and reproduction in any medium, as long as the original work is properly cited. ISBN: $978-81-936820-0-5$ 


\section{LITERATURE SURVEY}

The literature review plays a very important role in the research process. It is a source from where research ideas are drawn and developed into concepts and finally theories. It also provides the researcher a bird's eye view about the research done in that area so far. Depending on what is observed in the literature review, a researcher will understand where his/her research stands. Here in this literature survey, all primary, secondary and tertiary sources of information were searched.

The method of monitoring patient, pulse rate, body temperature has interacted jointly and communicate to one another to produce raw data that is passed through efficient protocol. In this interact method which is helpful to avoid risk. IoT is way to communicate all sensors efficiently together and monitor all data in real time in nature.

In this paper, the information gathering innovations that sense changes in physical status of things for then put away and shared this data. IoT utilizing diverse correspondence designs demand/reaction and notice in things with Nano-innovation that will supply and convey and cooperate with a specific and goal to fulfill some primary reason and client application. Interface regular articles and gadgets to internet with a practical framework distributing pieces of proof for example pulse rate sensor. Here the arrangement of database in cloud is used to permit the data store in large manner and separate the data easily. The method of monitoring patient, pulse rate, body temperature has interacted jointly and communicate to one another to produce raw data that is passed through efficient protocol. In this interact method which is helpful to avoid risk. Io'T is way to communicate all sensors efficiently together and monitor all data in real time in nature.

Here LM-35 temperature sensor is used to detect the patient body temperature, room temperature, humidity, pulse rate. The patient pulse will continuously monitor by pulse rate sensor. The chest belt around the sensor especially designed for the diagnosis of critical stage, of heart patient. The sensor which is connected to ventilation tube, which diagnosis the $\mathrm{co} 2$ level in the patient. There are many number of sensors and all the sensors are attached to the microcontroller. This will pass the result to the server and those data are saved for further processing. This is done with the implementation of MQTT protocol. In this paper the implementation work in wireless sensor networks. The data transmitted and received during the process lacks security.

In this paper, the process works in mobile networks as well as wireless sensor networks. This work is done and implementation of MQTT and its application with light weight IoT protocol for retrieval of sensor data. We are referring in this paper for using of android application.

Proceedings of the $3^{\text {rd }}$ National Conference on Image Processing, Computing, Communication, Networking and Data Analytics (NCICCNDA 2018) 


\section{PATIENT MONITORING SYSTEM}

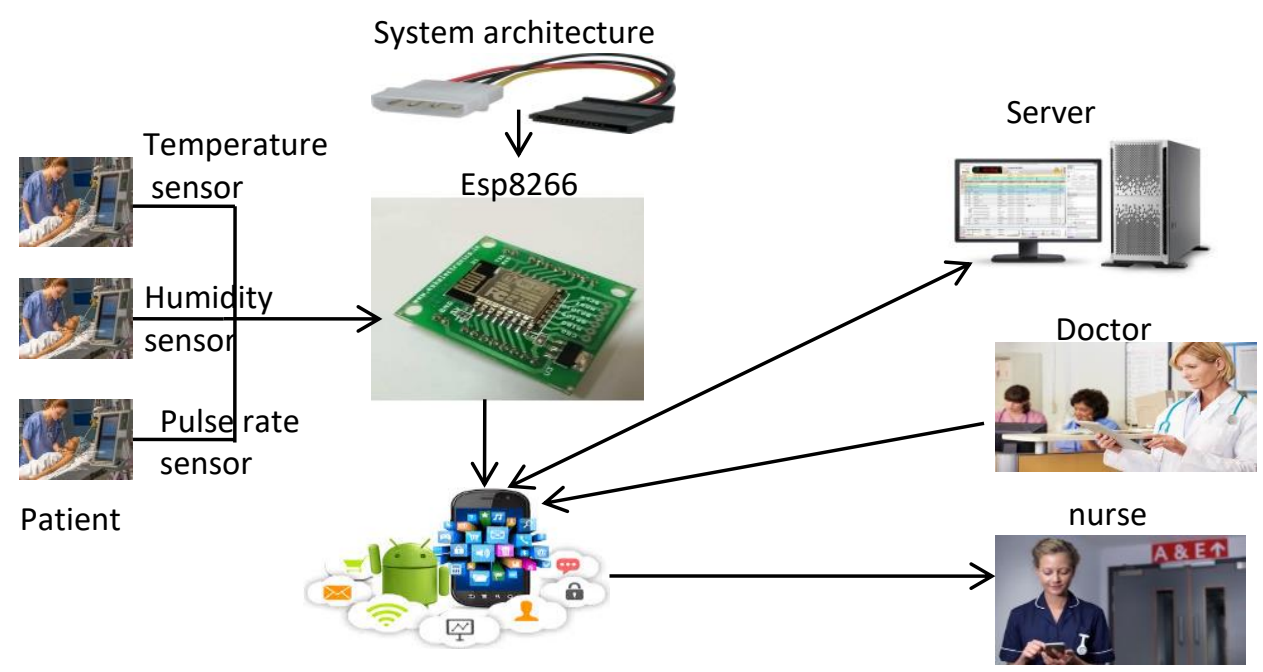

Android Application

Patient is monitored by the sensors mounted on them. server will host the information on the network. The server collects the data from the sensors. On another side doctors are acting as clients. If the doctors request server for data, the MQTT protocol helps to connect server with clients to display the current status and check for variation of sensor values. here the transferring sensor data from a room temperature, body temperature, humidity, pulse rate sensor to a control sensor. The sensors will be connected to a raspberry pi, which act as a gateway to MQTT broker, which resides in the cloud. on another side the control center, that also has an MQTT client and receivers the data. we will implement a notification, which alerts the center control if the sensor is disconnected. using android application doctors send the prescription and respective nurse receive the notification sending by the doctor and from the sensors if any variations are raised.

\section{CONCLUSION}

We presented to monitor the patient continuously to avoid from critical stage. We used Energy Efficient MQTT client-server protocol, to produce low power consumption at the cost of high latency. All the sensors are connected to the server with help of connected to the server with the help of MQTT protocol with high efficiency. From the received data, we can diagnosis the patient continuously with utmost care. If any critical stage obtained means, we can easily diagnosis and immediately alert message is sent to doctors and patient's take care. To diagnosis the patient Bayes machine learning technique is used perfectly for identifying abnormality in patient. From this paper, it is expecting to monitor the patient and to improve the patient care through Io'T. Io'T provides timely and cost-effective response to those critical 
situations. Here MQTT protocol performs good for patient monitoring in terms of latency, reliability and battery lifetime.

\section{References}

[1] Y. Li, L. Guo, C. Wu, C.-H. Lee, and Y. Guo, "Building a cloud-based platform for personal health sensor data management," in Biomedical and Health Informatics (BHI), 2014 IEEE-EMBS International Conference on, 2014, pp. 223-226

[2] Y. Yao and J. Gehrke, "The cougar approach to in-network query processing in sensor networks," ACM Sigmod Record, vol. 31, pp. 9-18, 2002

[3] D. J. Abadi, D. Carney, U. Çetintemel, M. Cherniack, C. Convey, S. Lee, et al., "Aurora: a new model and architecture for data stream management," The VLDB Journal-The International Journal on Very Large Data Bases, vol. 12, pp. 120-139, 2003

[4] M. Yuriyama and T. Kushida, "Sensor-cloud infrastructure-physical sensor management with virtualized sensors on cloud computing," in Network-Based Information Systems (NBiS), 2010 13th International Conference on, 2010, pp. 1-8

[5] C. A. Volgman, S. Wang, D. Mehta, N. Nazir, S. Alexander, K. Krishnan, et al., "O016 AliveCor Heart Monitoring: Is it a practical alternative to a traditional ECG monitor for a developing nation," Global Heart, vol. 9, pp. e4-e5, 2014

[6] D. Benitez, P. Gaydecki, A. Zaidi, and A. Fitzpatrick, "The use of the Hilbert transform in ECG signal analysis," Computers in biology and medicine, vol. 31, pp. 399-406, 2001

[7] P. De Chazal, M. O'Dwyer, and R. B. Reilly, "Automatic classification of heartbeats using ECG morphology and heartbeat interval features," Biomedical Engineering, IEEE Transactions on, vol. 51, pp. 1196-1206, 2004

[8] C. Ye, M. T. Coimbra, and B. V. Kumar, "Arrhythmia detection and classification using morphological and dynamic features of ECG signals," in Engineering in Medicine and Biology Society (EMBC), 2010 Annual International Conference of the IEEE, 2010, pp. 1918-1921.

[9] M. Engin, "ECG beat classification using neuro-fuzzy network," Pattern Recognition Letters, vol. 25, pp. 17151722,2004

[10] B. Babusiak and M. Gala, "Detection of Abnormalities in ECG," in Information Technologies in Biomedicine, ed: Springer, 2012, pp. 161-171

[11] B. Babusiak and M. Gala, "Detection of Abnormalities in ECG," in Information Technologies in Biomedicine, ed: Springer, 2012, pp. 161-171

[12] A. Lourenço, H. Silva, and C. Carreiras, "Outlier detection in nonintrusive ECG biometric system," in Image Analysis and Recognition, ed: Springer, 2013, pp. 43-52

[13] Y. Li, L. Guo, C. Wu, C.-H. Lee, and Y. Guo, "Building a Cloud-Based Platform for Personal Health Sensor Data Management," presented at the IEEE-EMBS International Conferences on Biomedical and Health Informatics, Valencia, Spain, 2014

[14] Y. Li, L. Guo, and Y. Guo, "An Efficient and Performance-Aware Big Data Storage System," in Cloud Computing and Services Science, ed: Springer, 2013, pp. 102-116

[15] Y. Li, L. Guo, A. Supratak, and Y. Guo, "Enabling Performance as a Service for a Cloud Storage System," presented at the 7th IEEE International Conference on Cloud Computing, Alaska, USA, 2014

[16] A. L. Goldberger, L. A. Amaral, L. Glass, J. M. Hausdorff, P. C. Ivanov, R. G. Mark, et al., "Physiobank, physIotoolkit, and physionet components of a new research resource for complex physiologic signals," Circulation, vol. 101, pp. e215-e220, 2000 